\title{
A Biochemical Analysis of Patients with COVID-19 Infection
}

\author{
Adil R. Sarhan $(\mathbb{D}$, Thaer A. Hussein $(\mathbb{D}$, Mohammed H. Flaih $\mathbb{D}$, and Khwam R. Hussein \\ Department of Medical Laboratory Techniques, Nasiriyah Technical Institute, Southern Technical University, \\ Nasiriyah 64001, Iraq \\ Correspondence should be addressed to Adil R. Sarhan; ar.sarhan@stu.edu.iq
}

Received 16 August 2021; Revised 21 September 2021; Accepted 5 October 2021; Published 22 October 2021

Academic Editor: Saad Tayyab

Copyright ( 92021 Adil R. Sarhan et al. This is an open access article distributed under the Creative Commons Attribution License, which permits unrestricted use, distribution, and reproduction in any medium, provided the original work is properly cited.

Several studies have demonstrated that age, comorbidities, and abnormalities in different clinical biomarkers can be important to understand disease severity. Although clinical features of COVID-19 have been widely described, the assessment of alterations of the most common biochemical markers that are reported in patients with COVID-19 still has not been well established. Here, we report clinical and blood biochemical indicators of 100 patients with COVID-19. Throat-swab upper respiratory samples were obtained from patients and real-time PCR was used to confirm SARS-CoV-2 infection. Gender, age, and clinical features such as diabetes mellitus, hypertension, and smoking habits were investigated. Biochemical parameters were categorized and analyzed according to these clinical characteristics. Triglycerides, GPT, and ALP are the biochemical markers that changed the most in the group of hypertension patients. Cholesterol and triglycerides were significantly different $(P=0.01 ; P=0.04$, respectively) between diabetic and nondiabetic patients with COVID-19. Potassium levels were significantly different $(P=0.03)$ when comparing smokers with nonsmoker patients. Our results suggest several potential biochemical indexes that changed in patients with COVID-19 and whether certain comorbidity and clinical characteristics influence these markers.

\section{Introduction}

Coronavirus disease 2019 (COVID-19) is a contagious disease caused by the recently discovered severe acute respiratory syndrome coronavirus 2 , which is still spreading throughout the world. Most infected patients with COVID19 will have mild to moderate respiratory illness that will be recovered without special medical care. However, elderly people and those with chronic medical conditions such as respiratory disease, heart disease, diabetes, and cancer are more likely to develop severe illnesses [1-3]. According to recent World Health Organization (WHO) figures, more than 110 million cases have been confirmed worldwide, with 2.44 million deaths as of February 16, 2021 [4].

Iraq has been greatly impacted by the ongoing outbreak of COVID-19. The first COVID-19 patient was reported in Iraq on 24 February 2020 [5]. Currently, as of February 16, 2021, over 650,000 diagnosed cases have been reported with more than 13,000 deaths [4]. Several biochemical tests are dramatically changed in patients with COVID-19. Early studies of COVID-19 showed substantial levels of alanine aminotransferase (ALT) in intensive care unit patients [6]. In addition, D-dimer, creatinine, blood urea, and neutrophil levels were also substantially increased in patients with severe symptoms, while lymphocyte counts were reduced [7]. D-dimer levels were also elevated in $70 \%$ of patients with severe symptoms and deceased cases [8].

Deng et al. found that, in certain patients, ALT and aspartate transaminase (AST) showed higher levels than the normal range with decreased total bilirubin levels. Creatinine and creatine phosphokinase were both increased in $13 \%$ of patients with COVID-19 [9] while Wu et al. observed a change in the percentages of liver function biomarkers (ALB, GGT, AST, ALT, TBIL, and ALP) [10].

This study aimed to demonstrate a systematic assessment for some of the biochemical laboratory tests in patients with COVID-19. All patients were admitted to the Al-Hussein Teaching Hospital, Thi-Qar Province, Iraq. Real-time PCR was used to confirm SARS-CoV-2 infection. Clinical characteristics and blood biochemical tests of COVID-19 patients were examined and recorded. 


\section{Materials and Methods}

2.1. Data Collection. All patients were referred to Al-Hussein Teaching Hospital in Thi-Qar Province, Iraq, between November 2 and December 30, 2020, showing COVID-19 symptoms. Throat-swab upper respiratory specimens were obtained from 100 patients and real-time PCR (polymerase chain reaction) was used to confirm SARS-CoV-2 infection. Clinical characteristics and blood biochemical tests of COVID-19 patients were examined and recorded. Gender, age, and clinical characteristics such as diabetes mellitus, hypertension, and smoking have been investigated. Informed consent was obtained from patients. The study has been approved by the Institutional Review Board.

2.2. Sample Collection and Data Processing. Venous blood $(4.5 \mathrm{~mL})$ was obtained. Blood samples were dispensed into a gel tube. All tubes were allowed to stand for 30 minutes at room temperature, followed by centrifugation for $10 \mathrm{~min}$ utes at $3500 \mathrm{rpm}$ to get the serum. Liver and kidney function tests including alanine transaminase (ALT), aspartate aminotransferase (AST), alkaline phosphatase (ALP), total bilirubin, creatinine, and blood urea were measured. Uric acid, triglyceride, total cholesterol, high-density lipoprotein (HDL), calcium $\left(\mathrm{Ca}^{+2}\right)$, sodium $\left(\mathrm{Na}^{+}\right)$, potassium $\left(\mathrm{K}^{+}\right)$, chloride $\left(\mathrm{Cl}^{-}\right)$, magnesium $\left(\mathrm{Mg}^{+2}\right)$, and phosphorus $(\mathrm{P})$ were also measured using Gesan Chem-200 platform (Gesan Production SRL, Italy) according to the manufacturing protocols.

2.3. Statistical Analysis. Biochemical parameters were analyzed and categorized according to the following variables: (a) diabetes mellitus, (b) blood pressure, (c) smoking, and (d) gender. Biochemical tests were compared across patients grouped in these categories. Significance testing was performed in GraphPad Software using a $t$-test. A $P$ value of $<0.05$ indicated statistical significance.

\section{Results}

A total of 100 COVID-19 patients were admitted to AlHussein Teaching Hospital in Thi-Qar Province, Iraq, from 2 November to 30 December 2020. Most cases (52\%) were between the ages of 21 and 40 years, followed by those aged 41-60 years (29\%). Approximately $54 \%$ of patients were female and $46 \%$ were male (Table 1 ). Most cases experienced influenza-like symptoms such as fever, cough, and mild myalgia during their time at the hospital. All patients were discharged following recovery of clinical symptoms. Diabetes mellitus, present in 23 out of 100 patients, was the most common comorbidity, followed by blood pressure, present in 14 patients. In addition, a summary of biochemical indexes outcomes among patients with COVID-19 is presented in Table 2.

In the blood pressure group, triglycerides, alanine aminotransferase (GPT), and alkaline phosphatase (ALP) were the most common biochemical laboratory abnormalities identified (Figure 1). However, no significant association was found between elevated blood pressure and normal blood pressure in all biochemical laboratory parameters as shown in Figure 1. We then analyzed the correlation between the biochemical characteristics and diabetes mellitus in patients with COVID-19. Among the biochemical parameters, cholesterol and triglycerides had a significant difference $(t=2.572, P=0.01 ; t=1.992, P=0.04$, respectively) between the diabetic and nondiabetic COVID19-infected patients (Figure 2). Furthermore, in a few patients, creatinine, alanine aminotransferase (GPT), alkaline phosphatase (ALP), and aspartate aminotransferase (GOT) levels were shown to be higher than the normal range. However, when comparing them according to diabetic and nondiabetic classification, none of these variations were statistically significant (Figure 2).

Figure 3 indicates the gender-based breakdown of biochemical indexes. Out of 100 patients, 54\% of the patients were female and $46 \%$ were male. Some biochemical measures such as creatinine, triglycerides, alkaline phosphatase and GOT, were found to have increased levels (Figure 3). However, when comparing these biomarkers between males and females, no evidence of significant differences was found.

Smoking has been shown to increase serum lipid profiles including triglycerides $[11,12]$. We, therefore, analyze whether smoking habits may influence the balance of serum biochemistry in patients with COVID-19. Low serum concentrations of magnesium, phosphorus, and calcium, have been seen in certain patients (Figure 4). Further analysis revealed high serum levels of creatinine, GPT, ALP, GOT, and urea in just a few other patients (Figure 4) whereas only potassium concentrations were significantly different $(t=2.140, P=0.03)$ when comparing smokers with nonsmoker patients.

\section{Discussion}

COVID-19 is an ongoing pandemic and the virus is still spreading worldwide. As stated by World Health Organization (WHO) reports, more than 110 million cases have been confirmed globally and 2.44 million deaths through February 16, 2021 [4]. On 24 February 2020, the first COVID-19 patient was confirmed in Iraq [5]. Recently, as of February 16, 2021, over 650,000 diagnosed cases have been reported with over 13,000 deaths [4]. Several studies have shown that age, comorbidities, and abnormalities of various clinical biomarkers can be essential to understand disease severity [13-16]. Even though clinical features of COVID-19 have been widely described, the overview of changes in the most common biochemical parameters that are observed in patients with COVID-19 infection is still unclear. Therefore, this report aims to study the changes in certain biochemical markers encountered in patients with COVID-19. In addition, clinical characteristics and comorbidity in 100 patients with COVID-19 have also been studied.

A strong relationship between lipid profiles and hypertension has been reported in [17-20]. Higher triglycerides values were found in patients with elevated blood pressure which are consistent with these reports. We found 
TABLe 1: Biochemical characteristics of patients with COVID-19 $(N=100)$.

\begin{tabular}{|c|c|c|c|c|c|c|c|c|c|c|c|c|c|c|c|c|c|c|c|c|c|c|}
\hline ID & $\mathrm{Ca}$ & $\mathrm{Na}$ & $\mathrm{K}$ & $\mathrm{Cl}$ & $\mathrm{Mg}$ & $\mathrm{P}$ & Urea & Crea. & UA & Chol. & Tri & HDL & GOT & GPT & ALK & TSB & $\mathrm{D}$ & $\mathrm{BP}$ & $S$ & $\mathrm{C}$ & $\mathrm{G}$ & Age \\
\hline 1 & 97 & 39 & .1 & 99 & 1.9 & 3.2 & 29 & 0.7 & 4.8 & 178 & 134 & 32 & 26 & 32 & 178 & 0.3 & No & Jo & No & No & $\mathrm{F}$ & 35 \\
\hline 2 & 2.16 & 41 & 4 & 100 & 1.9 & 2.5 & 28 & & 5.2 & & 125 & & & & 06 & 0.4 & No & No & No & No & $\mathrm{F}$ & 19 \\
\hline 3 & 2.42 & 38 & 3.9 & 99 & 1.8 & 2.6 & 30 & & 4.4 & 210 & 166 & & & & 167 & 0.3 & No & No & No & No & $\mathrm{M}$ & 45 \\
\hline 4 & 2.46 & 144 & 3.9 & 102 & 2 & 2.7 & 29 & 0.7 & 4.2 & 156 & 156 & 43 & & & 56 & 0.2 & Yes & No & No & No & $\mathrm{F}$ & 29 \\
\hline 5 & 2.33 & 137 & 4 & 101 & 1.8 & 2.8 & 33 & 0.8 & 4.9 & 188 & 145 & 45 & בר & 32 & 166 & 0.2 & No & No & No & No & $\mathrm{F}$ & 33 \\
\hline 6 & 2.4 & 138 & 3.8 & 99 & 2.1 & 3.3 & 32 & 0.8 & 3.9 & 149 & 234 & 32 & & & 145 & 0.3 & No & No & No & No & $\mathrm{F}$ & 20 \\
\hline 7 & 2.25 & 140 & 4.1 & 98 & 2 & 2.6 & 41 & & 5.6 & 154 & 198 & 5 & & & 345 & 0.7 & No & No & No & No & $\mathrm{M}$ & 41 \\
\hline 8 & 2.3 & 143 & 4.4 & 99 & 1.2 & 3.3 & 37 & - & 5.1 & 177 & 198 & & & & 89 & 0.6 & No & No & No & No & $\mathrm{F}$ & 38 \\
\hline 9 & 2.43 & 141 & 4.1 & 99 & 1.9 & 3.5 & 27 & 0.6 & 3.8 & 182 & 167 & 51 & 24 & 27 & 156 & 0.3 & No & Yes & No & No & $\mathrm{M}$ & 19 \\
\hline 10 & 2.5 & 144 & 4.2 & 98 & 1.9 & 3.2 & 38 & 1.4 & 4.6 & 163 & 156 & 46 & & & 176 & 0.4 & Yes & No & No & No & $\mathrm{F}$ & 44 \\
\hline 11 & 2.35 & 145 & 4 & 100 & 1.8 & 2.9 & 36 & 1.2 & 4.8 & 148 & 167 & 39 & & & 145 & 0.3 & No & No & No & No & $\mathrm{M}$ & 39 \\
\hline 12 & 2.39 & 143 & 4.3 & 99 & 2 & 4.2 & 29 & 0.7 & 3.8 & 198 & 233 & & & & 148 & 0.2 & No & No & No & No & $\mathrm{F}$ & 19 \\
\hline 13 & 2.42 & 144 & 3.9 & 98 & 2.1 & 3.6 & 29 & 0.7 & 3.7 & 202 & 256 & 35 & 24 & 41 & 198 & 0.5 & No & es & No & No & $\mathrm{F}$ & 18 \\
\hline 14 & 2.37 & 148 & 4 & 101 & 1.9 & 3.2 & 28 & 0.6 & 4 & 139 & 167 & 4 & & 5 & 356 & 0.8 & No & No & No & No & $\mathrm{F}$ & 23 \\
\hline 15 & 2.32 & 141 & 3.8 & 102 & 2.1 & 2.5 & 27 & 0.6 & 3.8 & 168 & 198 & & & & 398 & 0.8 & No & To & No & No & $\mathrm{M}$ & 27 \\
\hline 16 & 22 & 142 & 4.1 & 99 & 1.9 & 2.6 & 30 & & 4.1 & 190 & 216 & & & & 189 & 0.7 & Yes & To & & No & $F_{1}$ & 22 \\
\hline 17 & 2.2 & 139 & 4 & 98 & 1.8 & 2.7 & 30 & 0.9 & 4.5 & 175 & 198 & 5 & & & 69 & 0.4 & No & To & No & Jo & 1 & 30 \\
\hline 18 & 2.19 & 137 & 3.9 & 99 & 2 & 2.8 & 35 & 1 & 5.5 & 16 & 189 & 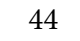 & & 2 & 144 & 0.3 & No & No & No & No & $\mathrm{M}$ & 52 \\
\hline 19 & 2.31 & 139 & 3.9 & 100 & 2.2 & 3.3 & 29 & 0.6 & 3.7 & & 215 & 3 & & & 54 & 0.4 & No & & & No & $\mathrm{F}$ & 42 \\
\hline 20 & 25 & 141 & 4 & 101 & 2.1 & 2.6 & 25 & & & & & & & & 89 & 0.7 & No & es & Yes & No & $\mathrm{M}$ & 34 \\
\hline 21 & 2.1 & 138 & 3.8 & 102 & 1.9 & 3.3 & 35 & 1 & 5. & 16 & 177 & . & & & 8 & 0.5 & No & o & & No & $\mathrm{F}$ & 52 \\
\hline 22 & 2 & 144 & 4.1 & 103 & 1.9 & 3.5 & 44 & 13 & 5.7 & & 155 & $\tau$ & & & 349 & 0.8 & No & To & No & Yes & $\mathrm{M}$ & 23 \\
\hline 23 & 2.29 & 137 & 4.4 & 102 & 1.8 & 3.2 & 2 & 0 & 4 & & 1 & 4 & & & & 0.9 & No & To & Yes & No & $\mathrm{M}$ & 36 \\
\hline 24 & 52 & 138 & 4.1 & 101 & 2 & 2.9 & 2 & & 3.6 & & & & & & & 1 & Yes & & No & No & $\Gamma$ & 51 \\
\hline 25 & 2.37 & 140 & 4.2 & 99 & 1.8 & 4.2 & 3 & & 4 & & 178 & & & & & 0.6 & No & & & es & 2 & 28 \\
\hline 26 & 197 & 143 & 4 & 99 & 2.1 & 3.6 & 4 & & 5 & & 197 & & & & & 0.9 & No & & No & No & $\mathrm{M}$ & 74 \\
\hline 27 & 2.16 & 141 & 4.3 & 100 & 2 & 3.2 & 4 & 1 & 5.9 & & 1 & 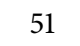 & & & & 1.1 & Yes & & No & No & $\mathrm{F}$ & 53 \\
\hline 28 & 2.42 & 144 & 3.9 & 99 & 1.2 & 2.5 & 3 & & 4.4 & & 1 & & & & 44 & & No & & Yes & No & $\mathrm{M}$ & 22 \\
\hline 29 & 2.46 & 145 & 4 & 102 & 1.9 & 2.6 & 4 & 1. & 6 & & & & & & & & Yes & & No & Jo & $\mathrm{F}$ & 54 \\
\hline 30 & 233 & 143 & 3.8 & 101 & 1.9 & 2.7 & 44 & & 5.7 & & & & & & & 0.2 & No & & No & No & $\mathrm{M}$ & 37 \\
\hline 31 & 2.4 & 144 & 4.1 & 99 & 1.8 & 2.8 & 30 & & 4.3 & & & & & & 1 & 0.2 & No & & No & No & $\mathrm{F}$ & 25 \\
\hline 32 & 2.2 & 148 & 4 & 98 & 2 & 3.3 & 3 & & & & & & & & & & & & & To & & 7 \\
\hline 33 & 2 & 141 & 3.9 & 99 & 2.1 & 2.6 & 2 & & 3 & & 186 & & & & & & No & 0 & & es & 2 & 0 \\
\hline 34 & 2.43 & 142 & 3.9 & 99 & 1.9 & 3.3 & $2 S$ & & 4.6 & & 216 & & & & 9 & 0.4 & No & To & No & No & $\mathrm{M}$ & 55 \\
\hline 35 & & 139 & 4 & 98 & 2.1 & 3.5 & 2 & & 5 & & & & & & & 0.5 & Yes & & & No & $\mathrm{M}$ & 46 \\
\hline 36 & ? & 137 & 3.8 & 100 & 1.9 & 3. & 0 & & & & & & & & & & No & & & No & $\mathrm{F}$ & 36 \\
\hline 37 & 2.39 & 139 & 4.1 & 99 & 1.8 & 2.9 & 3 & & 4 & & 1 & & & & 2 & 0.7 & No & 0 & & No & $\mathrm{M}$ & 34 \\
\hline 38 & 2.42 & 141 & 4.4 & 98 & 2 & 4.2 & 34 & & 5.2 & & & & & & & 0.4 & Yes & 0 & No & No & $\mathrm{F}$ & 18 \\
\hline 39 & 2.37 & 138 & 4.1 & 101 & 2.2 & 3.6 & 41 & & 5.9 & & & & & & & 0.3 & No & & No & No & $\mathrm{F}$ & 43 \\
\hline 40 & 2.32 & 144 & 4.2 & 102 & 2.1 & 3.2 & 3 & 1 & 6. & & 2 & 2 & & & & 0.5 & No & & & No & $\mathrm{M}$ & 55 \\
\hline 41 & 2.2 & 137 & 4 & 99 & 1.9 & 2.5 & $2 S$ & & 3.7 & 1 & 1 & 5 & & & 90 & 0 & Yes & 0 & & No & M & 26 \\
\hline 42 & 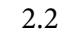 & 138 & 4.3 & 98 & 1.9 & 2.6 & 3 & & 5. & & 1 & & & & 3 & 0.3 & No & To & & No & $\mathrm{M}$ & 65 \\
\hline 43 & 2.19 & 140 & 3.9 & 99 & 1.8 & 2.7 & 2 & & & & & & & & & 0.3 & Yes & No & No & No & $\mathrm{F}$ & 26 \\
\hline 44 & 2.31 & 143 & 4 & 100 & 2 & 2.8 & 3 & & 4 & & & & & & & & No & o & & No & $\mathrm{F}$ & 14 \\
\hline 45 & 2 & 141 & 3.8 & 101 & 1.8 & 3.3 & 2 & & 4. & 1 & 1 & 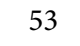 & & & , & 0 & No & No & No & No & 1 & 34 \\
\hline 46 & 2.1 & 144 & 4.1 & 102 & 2.1 & 2.6 & 28 & & 3.7 & 2 & 2 & 4 & & & 423 & 0.8 & No & No & Yes & No & $\mathrm{M}$ & 22 \\
\hline 47 & 2 & 145 & 4 & 103 & 2 & 3.3 & 4 & & & & & & & & & 0.9 & Yes & & & No & $\mathrm{M}$ & 31 \\
\hline 48 & 20 & 143 & & 102 & 1.2 & 3 & 3 & & 4 & & & & & & & 0.4 & No & & & No & $\mathrm{F}$ & 33 \\
\hline 49 & & 144 & 3.9 & 101 & 1.9 & 3. & . & & 6.6 & & & & & & & & No & & & No & $\mathrm{M}$ & 48 \\
\hline 50 & 2.37 & 148 & 4 & 99 & 1.9 & 2.9 & 29 & 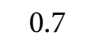 & 4 & 199 & 231 & 4 & 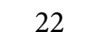 & 2 & 124 & 0.4 & No & No & No & No & $\mathrm{F}$ & 29 \\
\hline 51 & 1.97 & 141 & 3.8 & 99 & 1.8 & 4.2 & 34 & & 4.3 & & 22 & & & & & 0.3 & Yes & No & Yes & No & $\mathrm{M}$ & 46 \\
\hline 52 & 2.16 & 142 & 4.1 & 100 & 2 & 3.6 & 3 & & 5. & & 2 & & & & 260 & 0.8 & No & & No & No & $\mathrm{F}$ & 70 \\
\hline 53 & 2.42 & 139 & 4.4 & 99 & 2.1 & 3.2 & 3 & & 5. & & & & & & & 0 & No & & & Yes & $\mathrm{M}$ & 24 \\
\hline 54 & 2.46 & 137 & 4.1 & 102 & 1.9 & 2.5 & 29 & & 4.6 & 14 & 175 & 38 & & & 390 & 0.7 & No & No & Yes & No & $\mathrm{M}$ & 38 \\
\hline 55 & 2.33 & 139 & 4.2 & 101 & 2.1 & 2.6 & 27 & & 3.9 & 187 & 190 & 42 & 23 & 19 & 128 & 0.3 & Yes & No & No & No & $\mathrm{F}$ & 52 \\
\hline 56 & 2. & 141 & 4 & 99 & 1.9 & 2.7 & 2 & 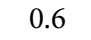 & 3. & 16 & 13 & 3 & & 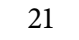 & 118 & 0.3 & No & No & No & No & $\mathrm{F}$ & 36 \\
\hline 57 & 2.25 & 138 & 4.3 & 98 & 1.8 & 2.8 & 33 & 1 & 5. & 16 & 14 & 3 & & 2 & 143 & 0.4 & No & No & Yes & No & $\mathrm{M}$ & 66 \\
\hline 58 & 2.3 & 144 & 3.9 & 99 & 2 & 3.3 & 52 & 1.7 & 6.4 & 209 & 246 & 35 & 18 & 26 & 136 & 0.3 & Yes & No & No & No & $\mathrm{F}$ & 64 \\
\hline 59 & 2.43 & 137 & 4 & 99 & 2.2 & 2.6 & 36 & 0.9 & 4.8 & 172 & 147 & 44 & 15 & 21 & 128 & 0.3 & No & No & Yes & No & $\mathrm{M}$ & 17 \\
\hline 60 & 2.5 & 138 & 3.8 & 98 & 2.1 & 3.3 & 26 & 0.7 & 4 & 191 & 210 & 36 & 34 & 38 & 168 & 0.6 & No & No & No & No & $\mathrm{F}$ & 23 \\
\hline
\end{tabular}


TABLE 1: Continued.

\begin{tabular}{|c|c|c|c|c|c|c|c|c|c|c|c|c|c|c|c|c|c|c|c|c|c|c|}
\hline ID & $\mathrm{Ca}$ & $\mathrm{Na}$ & $\mathrm{K}$ & $\mathrm{Cl}$ & $\mathrm{Ig}$ & $\mathrm{P}$ & rea & rea. & UA & Chol. & Tri & HDL & GOT & GPT & ALK & TSB & D & BP & S & C & G & $A \xi$ \\
\hline 61 & 2.35 & 10 & 1 & 0 & 9 & 5 & 29 & 8 & 4.7 & 226 & 33 & 29 & 26 & 3 & 66 & 0.5 & No & No & Yes & No & M & 45 \\
\hline 62 & 39 & 13 & 4 & 99 & 1.9 & 2 & 30 & & 4.1 & & & 34 & & & 55 & 8 & es & No & & Jo & $\mathrm{F}$ & 0 \\
\hline 63 & 42 & 41 & 9 & 98 & 1.8 & 2.9 & 33 & & 5.2 & & 67 & 45 & 0 & & & 0.9 & lo & No & & No & $\Gamma$ & 27 \\
\hline 64 & 37 & 44 & 9 & 01 & 2 & .2 & 32 & & 4.8 & & 198 & 20 & & & 87 & 0.7 & No & No & Yes & No & $M$ & 0 \\
\hline 55 & 32 & 45 & 4 & 102 & 1.8 & 6 & & & 7 & & 5 & 5 & & & 97 & 0.5 & No & No & No & Tes & M & 37 \\
\hline 56 & 2.2 & 143 & 3.8 & 99 & 2.1 & 3.2 & 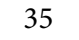 & & 6.6 & & 9 & 46 & & & 27 & .4 & es & No & & Jo & F & 40 \\
\hline 67 & 2.2 & 144 & .1 & 98 & 2 & 2.5 & 29 & & 4.9 & & 15 & 32 & & & & 0.3 & lo & No & & Jo & M & 26 \\
\hline 68 & 2.19 & 148 & 4.4 & 99 & 1.2 & 6 & 29 & & 4.3 & 18 & 190 & 43 & & & 54 & 0.5 & No & No & No & No & $\mathrm{F}$ & 24 \\
\hline 9 & 31 & 41 & .1 & 00 & 9 & & & & 8 & & & & & & & 8 & es & lo & es & Jo & M & 56 \\
\hline 0 & & 142 & .2 & 1 & 1.9 & 8 & 2 & & 6 & & 4 & & & & & & o & No & & To & M & 15 \\
\hline 71 & & 139 & 4 & 2 & 1.8 & 3 & 31 & & 5.3 & & 2 & & & & & 0.3 & o & No & No & Jo & M & 36 \\
\hline 72 & 2 & 137 & 4.3 & 3 & 2 & 2. & 3 & & 4.7 & 2 & & & & & & 0 & es & lo & NO & To & $\mathrm{F}$ & 21 \\
\hline 3 & & 39 & 9 & 2 & 2.1 & & & & & & & & & & & & o & lo & o & 0 & $\Gamma$ & 8 \\
\hline 74 & & 41 & 4 & 101 & 1.9 & & 4 & & 6.1 & & & & & & & & & lo & & o & $\mathrm{M}$ & 2 \\
\hline 75 & & 38 & 3.8 & 99 & 2.1 & 3.2 & 4. & & 6.4 & & & & & & & & 0 & lo & & 0 & & 16 \\
\hline 16 & & 44 & 4.1 & 99 & 1.9 & & 2 & & 4.4 & & & & & & & 0.4 & o & Yes & Yes & No & $\mathrm{M}$ & 77 \\
\hline 77 & 16 & 137 & 4 & 00 & 1.8 & & 3 & & 5.4 & & & & & & & 0 . & o & 0 & No & To & F & 57 \\
\hline 78 & 42 & 138 & & 99 & 2 & & & & 5 & & & & & & & & & 0 & & & M & 60 \\
\hline 79 & 6 & 40 & 9 & 102 & 2.2 & ? & 2 & & 35 & & & & & & & & Yes & lo & & es & M & 21 \\
\hline 80 & 33 & 143 & 4 & 101 & 2.1 & & 4 & & 6.2 & & & & & & & 0.7 & lo & Yes & Yes & Jo & $\mathrm{M}$ & 18 \\
\hline 81 & & 141 & & 99 & 1.9 & & 3 & 1 & 6 & & & & & & & 0 & & & & To & $\mathrm{F}$ & 10 \\
\hline 82 & 25 & 144 & & 98 & 1.9 & 2. & 2 & & 1 & & & & & & & & & 0 & & o & $\Lambda$ & 6 \\
\hline 83 & & 145 & & 99 & 1.8 & ? & 3 & & & & & & & & & & & [o & & Jo & M & 11 \\
\hline 84 & & 143 & & 99 & 2 & & 3 & & 4.9 & & & & & & & & & No & & & $F_{1}$ & 7 \\
\hline 85 & & 144 & & 98 & 1.8 & & & & & & & & & & & & & & & 0 & $\mathrm{~F}$ & 8 \\
\hline 86 & & 1 & 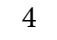 & 0 & 2.1 & & & & & & & & & & & & o & es & No & o & 1 & 3 \\
\hline 87 & & 141 & & 99 & 2 & & & & & & & & & & & & 0 & lo & Yes & Jo & $\mathrm{M}$ & 55 \\
\hline 88 & & 142 & & 98 & & & & & & & & & & & & & & & & No & $\mathrm{F}$ & 2 \\
\hline 89 & & & 4 & & & & & & & & & & & & & & & & & 0 & $\mathrm{~F}$ & 0 \\
\hline 90 & & 137 & & 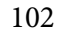 & .9 & 4 & 3 & & & & & & & & & & o & No & No & 0 & $\mathrm{~F}$ & 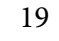 \\
\hline 91 & & 139 & 4.1 & 99 & 1.8 & 3. & 2 & & & & & & & & & & & lo & & Jo & $\mathrm{F}$ & 25 \\
\hline 92 & & 141 & 4 & 98 & 2 & & & & 4. & & & & & & & & No & Yes & No & No & $\mathrm{F}$ & 22 \\
\hline ? & & 138 & & 9 & 2.1 & & 30 & & & & & & & & & & Yes & Yes & & Jo & $\mathrm{M}$ & 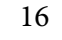 \\
\hline 94 & 2.31 & 144 & 3.9 & 100 & 1.9 & 2 & 3 & & & & & & & & & & No & No & & Jo & $\mathrm{F}$ & 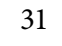 \\
\hline 95 & 2.5 & 137 & 4 & 101 & 2.1 & 2. & 20 & & & & & & & & & & & No & & No & $\mathrm{M}$ & 40 \\
\hline 96 & 2.1 & 138 & 8 & 02 & 1.9 & 2.8 & $2 \varepsilon$ & & 3. & & & 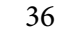 & & & & 0.5 & Yes & No & No & No & $\mathrm{F}$ & 41 \\
\hline$a^{\prime}$ & 2 & 1 & & & 1.8 & 3 & 38 & & 4 & & & & & & & & No & Yes & & To & $\mathrm{M}$ & 24 \\
\hline 98 & & 143 & .4 & 2 & 2 & ᄀ & 40 & & 6. & & & 4 & & & & 0.6 & No & No & & 0 & $\mathrm{M}$ & 55 \\
\hline 99 & 2.52 & 141 & 4.1 & 101 & 2.2 & 3.3 & 55 & & 6.5 & & & 45 & 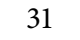 & & & 0.6 & No & No & & No & $\mathrm{F}$ & 59 \\
\hline 100 & 2.37 & 144 & 4.2 & 99 & 2.1 & 3.5 & 37 & 1.3 & 5.8 & 197 & 145 & 38 & 37 & 39 & 186 & 0.5 & Yes & Yes & No & No & $\mathrm{M}$ & 41 \\
\hline
\end{tabular}

Ca: calcium; Na: sodium; K: potassium; Cl: chloride; Mg: magnesium; P: phosphorus; Crea.: creatinine; Chol.: cholesterol; Trig: triglyceride; HDL: highdensity lipoprotein; GOT: aspartate aminotransferase; GPT: alanine aminotransferase; ALK: alkaline phosphatase; TSB; total bilirubin; D: diabetic; B: blood pressure; S: smoking; C: cancer; G: gender.

TABle 2: Comparison summary of biochemical parameters among patients with COVID-19 $(N=100)$.

\begin{tabular}{lc}
\hline Parameters & Mean \pm SD \\
\hline $\mathrm{Ca}, \mathrm{mmol} / \mathrm{L}$ & $2.31 \pm 0.15$ \\
$\mathrm{Na}, \mathrm{mmol} / \mathrm{L}$ & $141.25 \pm 2.94$ \\
$\mathrm{~K}, \mathrm{mmol} / \mathrm{L}$ & $4.04 \pm 0.17$ \\
$\mathrm{Cl}, \mathrm{mmol} / \mathrm{L}$ & $99.92 \pm 1.47$ \\
$\mathrm{Mg}, \mathrm{mg} / \mathrm{dL}$ & $1.92 \pm 0.2$ \\
$\mathrm{P}, \mathrm{mg} / \mathrm{dL}$ & $3.09 \pm 0.47$ \\
Urea, mg/dL & $33.27 \pm 6.3$ \\
Creatinine, mg/dL & $0.94 \pm 0.32$ \\
Uric acid, mg/dL & $4.86 \pm 0.87$ \\
Cholesterol, mg/dL & $176.18 \pm 24.68$ \\
Triglyceride, mg/dL & $186.56 \pm 37.67$ \\
$\mathrm{HDL}, \mathrm{mg} / \mathrm{dL}$ & $41.26 \pm 6.62$ \\
GOT, U/L & $32.28 \pm 12.86$ \\
GPT, U/L & $36 \pm 15.59$ \\
ALK, U/L & $192.72 \pm 90.59$ \\
TSB, mg/dL & $0.49 \pm 0.24$ \\
\hline
\end{tabular}



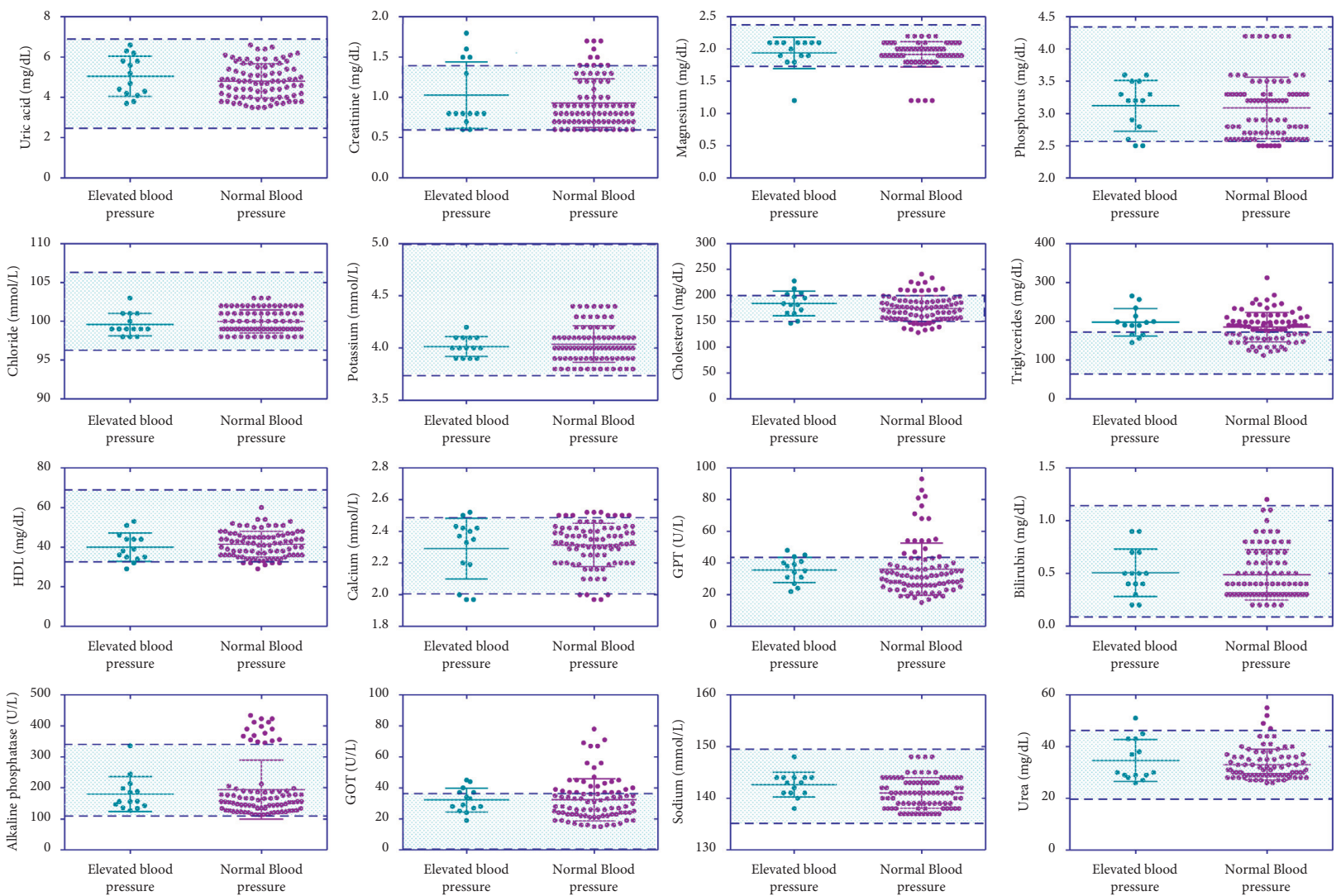

FIGURE 1: Scatter plots showing all the laboratory biochemical tests analyzed according to COVID-19 patients with normal blood pressure versus elevated blood pressure. The highlighted area indicates the normal range of each test.
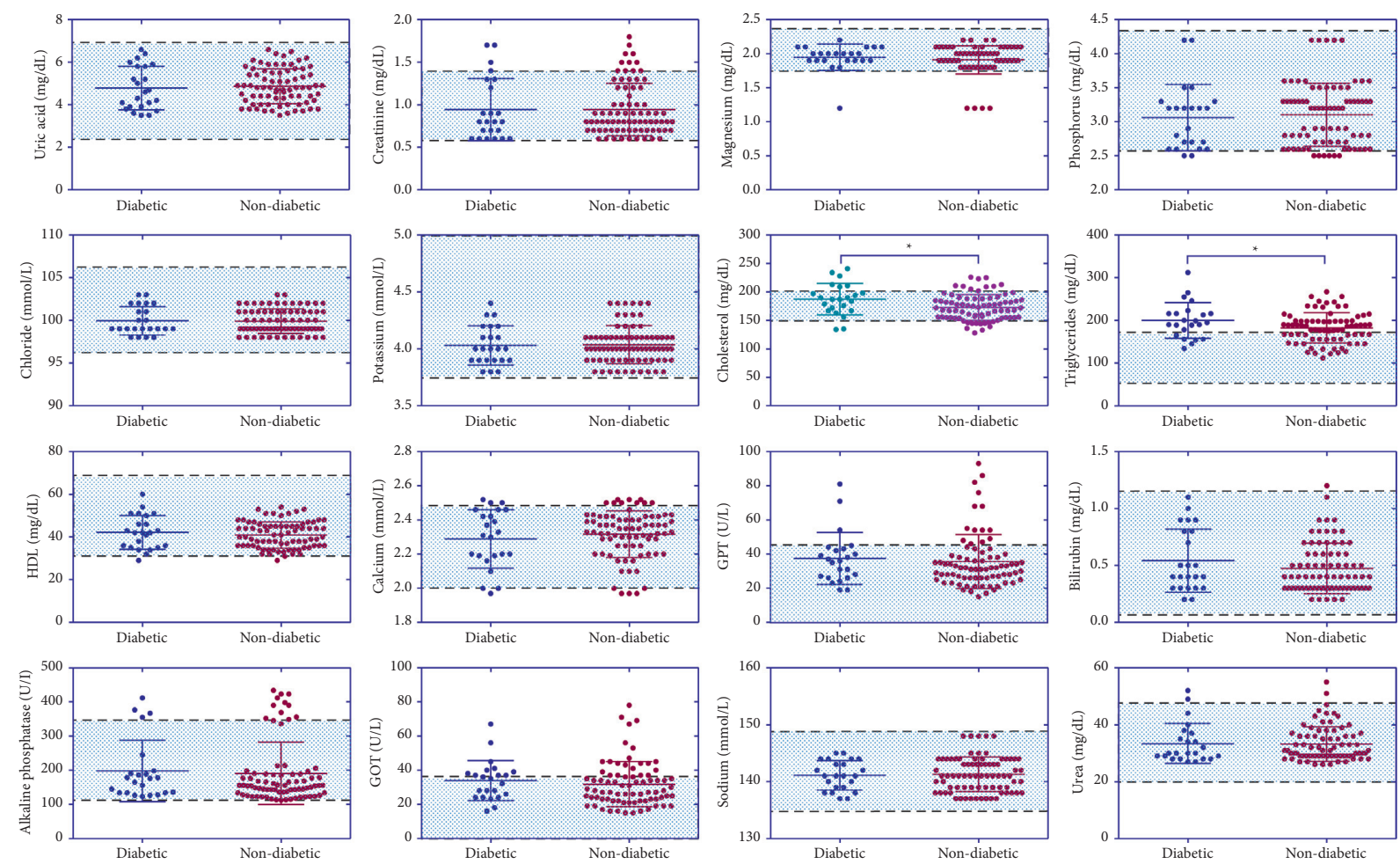

FIGURE 2: Scatter plots showing all the laboratory biochemical tests analyzed according to COVID-19 patients with diabetic versus nondiabetic. The highlighted area indicates the normal range of each test. Statistical significance was calculated by $t$-test $\left({ }^{*} P<0.05\right)$. 

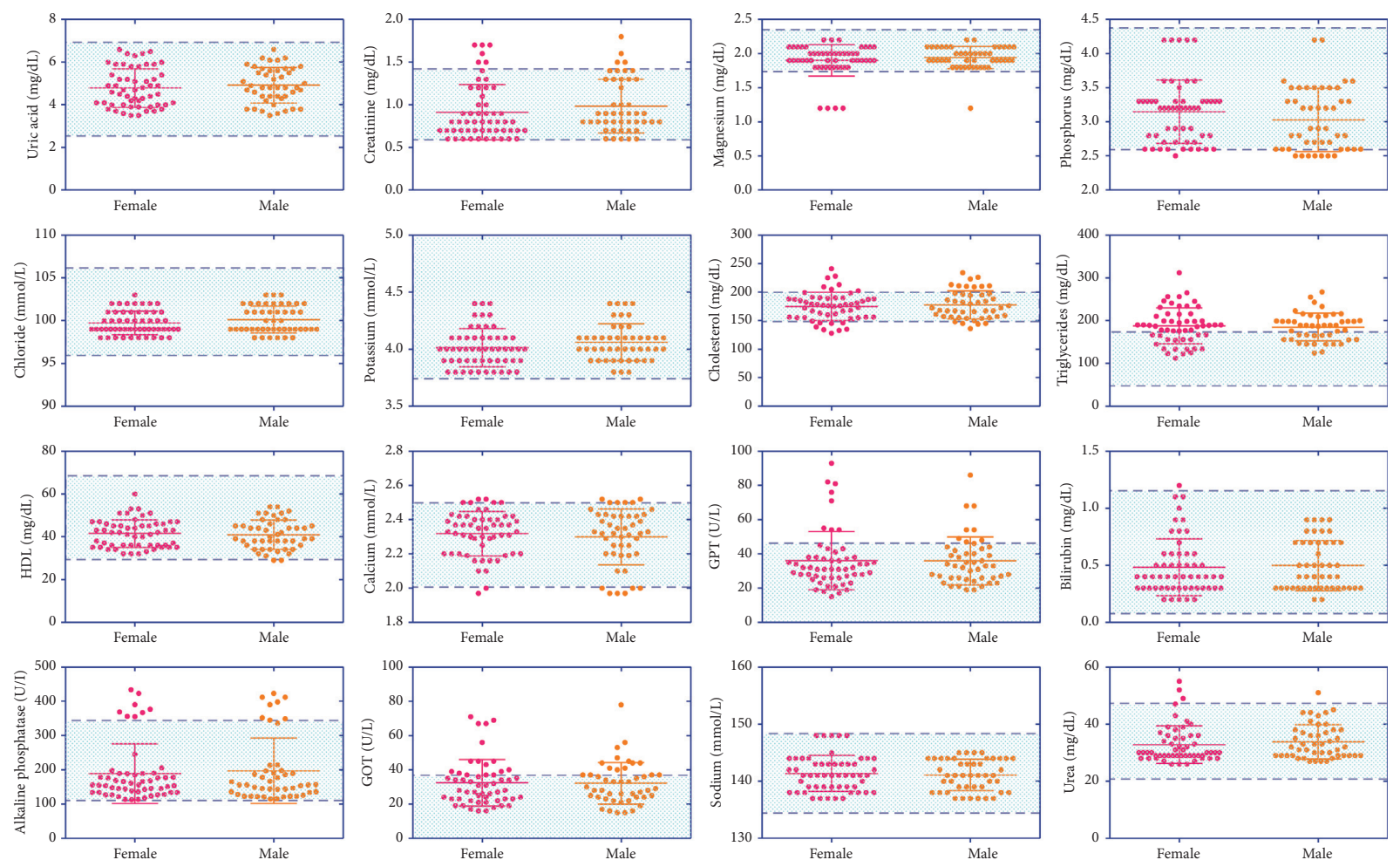

FIGURE 3: Scatter plots showing all the laboratory biochemical tests analyzed according to gender. The highlighted area indicates the normal range of each test.
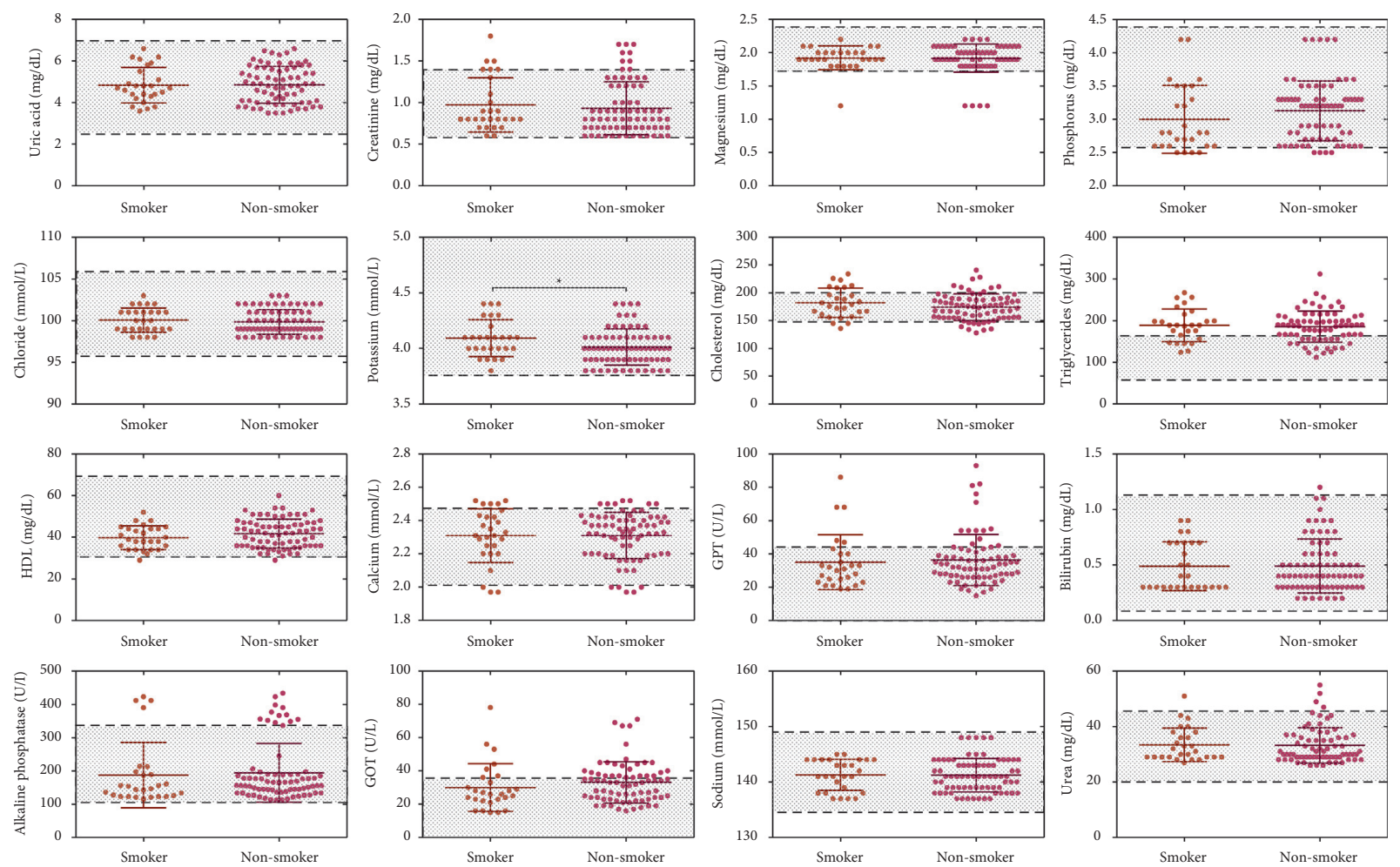

FIGURE 4: Scatter plots showing all the laboratory biochemical tests analyzed according to smoking habits. The highlighted area indicates the normal range of each test. Statistical significance was calculated by $t$-test $\left({ }^{*} P<0.05\right)$. 
that triglycerides, GPT, and GOT were elevated although there was no significant difference between COVID-19 patients with elevated and normal blood pressure suggesting that COVID-19 infection may alter these biochemical laboratory markers regardless of hypertension. Furthermore, the higher triglyceride levels in certain patients might be due to body fat and distribution, a condition not investigated in this study. These findings seem to be consistent with other studies which found that high levels of triglyceride were more positively correlated with body fat than with changes in blood pressure [21, 22].

We discovered a marked increase in levels of cholesterol and triglycerides in diabetic and nondiabetic COVID-19-infected patients. Creatinine, GPT, ALP, and GOT values were shown to be higher than the normal range in some COVID-19infected patients. The most notable comorbidities with COVID-19 in our study were diabetes (23\%) and hypertension (14\%). Coronaviruses bind to their target cells via angiotensinconverting enzyme 2 (ACE2), which is widely expressed in the kidney, intestine, and epithelial cells of the lung [23]. It has also been demonstrated that the expression of ACE2 is markedly upregulated in patients with diabetes which would promote the infection with COVID-19 [24, 25].

Several epidemiological data have shown no substantial correlation between smoking and disease severity in patients with COVID-19 [26-28]. In contrast, Leung et al. reported that smokers showed an upregulation of ACE2 gene expression than nonsmokers which facilitated COVID-19 infection [29]. When we analyzed the data according to smoking habits, elevated serum levels of triglyceride, creatinine, GPT, ALP, GOT, and urea were shown in few patients. Besides that, the concentrations of magnesium, phosphorus, and calcium were decreased in other patients. Interestingly, potassium levels showed significant differences when comparing smokers with nonsmoker patients. It has been shown that smoking habits might have induced alterations in potassium levels [30,31]. However, the link between smoking and potassium levels has not been well studied. In conclusion, our results suggest several potential biochemical indexes change in patients with COVID19 and that certain patient and clinical characteristics may influence these indexes.

\section{Data Availability}

All data are included in the supplementary materials.

\section{Disclosure}

The manuscript has been deposited as a preprint in Heliyon [32].

\section{Conflicts of Interest}

The authors declare no conflicts of interest.

\section{Acknowledgments}

The authors would like to acknowledge the cooperation and help they have received from Al-Hussein Teaching Hospital in Thi-Qar Province, Iraq.

\section{Supplementary Materials}

Overview of the clinical information, age, gender, and biochemical parameters for the 100 participants in the study. (Supplementary Materials)

\section{References}

[1] N. Zhu, D. Zhang, W. Wang et al., "A novel coronavirus from patients with pneumonia in China, 2019," New England Journal of Medicine, vol. 382, no. 8, pp. 727-733, 2020.

[2] J.-Y. Li, Z. You, Q. Wang et al., "The epidemic of 2019-novelcoronavirus (2019-nCoV) pneumonia and insights for emerging infectious diseases in the future," Microbes and Infection, vol. 22, no. 2, pp. 80-85, 2020.

[3] C. Huang, Y. Wang, X. Li et al., "Clinical features of patients infected with 2019 novel coronavirus in Wuhan, China," The Lancet, vol. 395, no. 10223, pp. 497-506, 2020.

[4] "Weekly epidemiological update - 16 February 2021, (n.d.)," 2021, https://www.who.int/publications/m/item/weeklyepidemiological-update---16-february-2021.

[5] A. R. Sarhan, M. H. Flaih, T. A. Hussein, and K. R. Hussein, Novel Coronavirus (COVID-19) Outbreak in Iraq: The First Wave and Future Scenario, Cold Spring Harbor Laboratory, Cold Spring Harbor, NY, USA, 2020.

[6] S.-R. Hao, S.-Y. Zhang, J.-S. Lian et al., "Liver enzyme elevation in coronavirus disease 2019: a multicenter, retrospective, cross-sectional study," American Journal of Gastroenterology, vol. 115, pp. 1-9, 2020.

[7] H. Parlakpinar and M. Gunata, "SARS-COV-2 (COVID-19): cellular and biochemical properties and pharmacological insights into new therapeutic developments," Cell Biochemistry and Function, vol. 39, no. 1, pp. 10-28, 2020.

[8] Z. Li, M. Wu, J. Yao et al., Caution on Kidney Dysfunctions of COVID-19 Patients, Cold Spring Harbor Laboratory, Cold Spring Harbor, NY, USA, pp. 1-25, 2020.

[9] X. Deng, B. Liu, J. Li, J. Zhang, Y. Zhao, and K. Xu, "Blood biochemical characteristics of patients with coronavirus disease 2019 (COVID-19): a systemic review and metaanalysis," Clinical Chemistry and Laboratory Medicine, vol. 58, no. 8, pp. 1172-1181, 2020.

[10] Y. Wu, H. Li, X. Guo et al., "Incidence, risk factors, and prognosis of abnormal liver biochemical tests in COVID-19 patients: a systematic review and meta-analysis," Hepatology International, vol. 14, no. 5, pp. 621-637, 2020.

[11] L. M. Håglin, B. Törnkvist, and L. O. Bäckman, "High serum phosphate and triglyceride levels in smoking women and men with CVD risk and type 2 diabetes," Diabetology \& Metabolic Syndrome, vol. 6, no. 1, 2014.

[12] M. Koda, I. Kitamura, T. Okura, R. Otsuka, F. Ando, and H. Shimokata, "The associations between smoking habits and serum triglyceride or hemoglobin A1c levels differ according to visceral fat accumulation," Journal of Epidemiology, vol. 26, no. 4, pp. 208-215, 2016.

[13] T. Riffe, E. Acosta, The COVID team et al., "Data Resource Profile: COVerAGE-DB: a global demographic database of COVID-19 cases and deaths," International Journal of Epidemiology, vol. 50, no. 2, pp. 390-390f, 2021.

[14] G. Lippi and M. Plebani, "Laboratory abnormalities in patients with COVID-2019 infection," Clinical Chemistry and Laboratory Medicine, vol. 58, no. 7, pp. 1131-1134, 2020.

[15] G. Lippi and E. J. Favaloro, "D-Dimer is associated with severity of coronavirus disease 2019: a pooled analysis," Thrombosis and Haemostasis, vol. 120, no. 05, pp. 876-878, 2020. 
[16] E. Urrechaga, U. Aguirre, P. P. España, and L. García de Guadiana, "Complete blood counts and cell population data from Sysmex XN analyser in the detection of SARS-CoV-2 infection," Clinical Chemistry and Laboratory Medicine, vol. 59, no. 2, pp. E57-E60, 2021.

[17] G. Ghooshchi, M. S. Yazdi, and M. P. Ramezan, Evaluation of the Lipid Profile of Hypertensive Patients Compared to Nonhypertensive Individuals, Mashhad University of Medical Sciences, Mashhad, Iran, 2014.

[18] V. Kotsis, S. Stabouli, S. Papakatsika, Z. Rizos, and G. Parati, "Mechanisms of obesity-induced hypertension," Hypertension Research, vol. 33, no. 5, pp. 386-393, 2010.

[19] A. Leone, "Editorial [hot topic: modifying cardiovascular risk factors: epidemiology and characteristics of hypertension-related disorders (executive guest editor: aurelio leone)]," Current Pharmaceutical Design, vol. 17, no. 28, pp. 2948-2954, 2011.

[20] A. Gebrie, N. Gnanasekaran, M. Menon, M. Sisay, and A. Zegeye, "Evaluation of lipid profiles and hematological parameters in hypertensive patients: laboratory-based crosssectional study," SAGE Open Medicine, vol. 6, Article ID 2050312118756663, 2018.

[21] M. Royo-Bordonada, C. Garcés, L. Gorgojo et al., "Saturated fat in the diet of Spanish children: relationship with anthropometric, alimentary, nutritional and lipid profiles," Public Health Nutrition, vol. 9, no. 4, pp. 429-435, 2006.

[22] R. O. Halperin, H. D. Sesso, J. Ma, J. E. Buring, M. J. Stampfer, and J. Michael Gaziano, "Dyslipidemia and the risk of incident hypertension in men," Hypertension, vol. 47, no. 1, pp. 45-50, 2006.

[23] Y. Wan, J. Shang, R. Graham, R. S. Baric, and F. Li, "Receptor recognition by the novel coronavirus from wuhan: an analysis based on decade-long structural studies of SARS coronavirus," Journal of Virology, vol. 94, no. 7, 2020.

[24] Z. Liu, X. Xiao, X. Wei et al., "Composition and divergence of coronavirus spike proteins and host ACE2 receptors predict potential intermediate hosts of SARS-CoV-2," Journal of Medical Virology, vol. 92, no. 6, pp. 595-601, 2020.

[25] X. C. Li, J. Zhang, and J. L. Zhuo, "The vasoprotective axes of the renin-angiotensin system: physiological relevance and therapeutic implications in cardiovascular, hypertensive and kidney diseases," Pharmacological Research, vol. 125, pp. 2138, 2017.

[26] G. Lippi and B. M. Henry, "Active smoking is not associated with severity of coronavirus disease 2019 (COVID-19)," European Journal of Internal Medicine, vol. 75, pp. 107-108, 2020.

[27] W. J. Guan, W. H. Liang, Y. Zhao et al., "Comorbidity and its impact on 1590 patients with COVID-19 in China: a nationwide analysis," European Respiratory Journal, vol. 55, 2020.

[28] W.-j. Guan, Z.-y. Ni, Y. Hu et al., "Clinical characteristics of coronavirus disease 2019 in China," New England Journal of Medicine, vol. 382, no. 18, pp. 1708-1720, 2020.

[29] J. M. Leung, C. X. Yang, A. Tam et al., "ACE-2 expression in the small airway epithelia of smokers and COPD patients: implications for COVID-19," European Respiratory Journal, vol. 55, no. 5, 2020.

[30] W. Sg, L. Af, S. Ag, and W. Ph, "Serum potassium, cigarette smoking, and mortality in middle-aged men," American Journal of Epidemiology, vol. 145, pp. 598-606, 1997.

[31] P. Padmavathi, V. D. Reddy, and N. Varadacharyulu, "Influence of chronic cigarette smoking on serum biochemical profile in male human volunteers," Journal of Health Science, vol. 55, no. 2, pp. 265-270, 2009.
[32] A. R. Sarhan, T. A. Hussein, M. H. Flaih, and K. R. Hussein, “A comprehensive analysis of biochemical indexes in patients with COVID-19 infection," SSRN Electronic Journal, 2021. 